\title{
Enantiomeric Separations of Chiral Pharmaceuticals using Chirally Modified Tetrahexahedral Au Nanoparticles
}

\author{
N. Shukla ${ }^{1}$, D. Yang ${ }^{2}$, A.J. Gellman ${ }^{2, *}$ \\ ${ }^{1}$ Institute of Complex Engineered Systems, \\ ${ }^{2}$ Department of Chemical Engineering, \\ Carnegie Mellon University, Pittsburgh, PA-15213, USA
}

\begin{abstract}
:
Tetrahexahedral (THH, 24-sided) Au nanoparticles modified with $D$ - or $L$-cysteine (Cys) have been used as enantioselective separators of the chiral pharmaceutical propranolol (PLL) in solution phase. Polarimetry has been used to measure the rotation of linearly polarized light by solutions containing mixtures of PLL and Cys/THH-Au NPs with varying enantiomeric excesses of each. Polarimetry yields clear evidence of enantiospecific adsorption of PLL onto the Cys/THH-Au NPs. This extends prior work using propylene oxide as a test chiral probe, by using the crystalline THH Au NPs with well-defined facets to separate a real pharmaceutical. This work suggests that chiral nanoparticles, coupled with a density separation method such as centrifugation, could be used for enantiomeric purification of real pharmaceuticals. A simple robust model developed earlier has also been used to extract the enantiospecific equilibrium constants for $R$ - and $S$-PLL adsorption onto the $D$ - and $L$-Cys/THH-Au NPs.
\end{abstract}

*Corresponding author: gellman@cmu.edu, 412-268-3848

(C) 2015. This manuscript version is made available under the Elsevier user license http://www.elsevier.com/open-access/userlicense/1.0/ 


\section{Introduction}

The two enantiomers of chiral pharmaceuticals have the same chemical structure but, nonetheless, have different pharmacological activity and toxicity. This creates an enormous market for enantiomerically pure pharmaceuticals and a critical need for enantioselective and enantiospecific chemical processes, because $\sim 50 \%$ of currently marketed pharmaceuticals are chiral $^{1-2}$. Most synthetic pharmaceuticals are prepared in racemic form (equimolar mixture of two enantiomers) and then require enantiospecific separation and purification. Enantioselective separations of synthetic chiral pharmaceuticals are, therefore, extremely important to reaching the full therapeutic potential of these compounds. The work described herein, demonstrates the potential for use of chiral nanoparticles (NPs) as adsorbent phases for enantioselective purification of chiral pharmaceuticals from solution phase ${ }^{3-5}$.

Prior work has shown that simple chiral probe molecules such as $R$ - and $S$-propylene oxide (PO) can be adsorbed enantioselectively onto the surfaces of Au NPs that have been chirally modified with either $D$ - or $L$-cysteine (Cys) ${ }^{6-8}$. The work herein, advances prior accomplishments by using tetrahexahedral (THH, 24-sided) Au NPs exposing well-defined crystal planes and, more importantly, demonstrating that the methodology works with use of a known pharmaceutical, propranolol (PLL). The value of NPs exposing single crystalline facets is that they have the potential to be more highly enantioselective that previously used spherical NPs exposing an inhomogeneous distribution of surface orientations. PLL hydrochloride (chemical structure in Figure 1D) is an example of a $\beta$-blocking drug used to treat high blood pressure and heart disease. It also demonstrates enantiospecific therapeutic impact ${ }^{1-2}$. S-PLL is 100 times more effective than $R$-PLL. Similarly, $S$-ibuprofen is 100 times more effective as an anti-inflammatory drug than its $R$-enantiomer ${ }^{1-2}$. The toxicology of other chiral pharmaceuticals reveals that the two enantiomers can have completely opposite pharmacological impact; one can be therapeutic while the other is toxic. Well known examples are thalidomide and dopamine. $L$ dopamine can be used to treat Parkinson's disease whereas $D$-dopamine is highly toxic ${ }^{2}$. Similarly, $S$-thalidomide can treat morning sickness in pregnant women whereas $R$-thalidomide is linked to birth defects ${ }^{1}$. Due to the differences in the pharmacological activity of enantiomers, the pharmaceutical industry takes enormous precautions to isolate enantiomerically pure materials for marketing as pharmaceuticals. 
Two widely used enantiomeric separations techniques used in the pharmaceutical industry are chiral high performance liquid chromatography and simulated moving bed chromatography ${ }^{2}$, ${ }^{9}$. Both of these techniques require chiral selectors which are either stationary or mobile and the choice of the most effective chiral column materials for any given separation is extremely important but also extremely difficult. In addition to the composition of the chiral stationary or mobile phase, the length of the column, and other environmental additions such temperature need to be optimized for any given separation.

There have been a variety of studies of the chiral structures and enantiospecific properties of chiral NPs ${ }^{3-5,10}$. In past work, we have developed the use of polarimetry as a tool for detection of the enantiospecific partitioning of chiral probe compounds such as propylene oxide (PO) between a solution phase and the surfaces of chiral Au NPs ${ }^{6-8}$. The key observation is that when a racemic mixture of $\mathrm{PO}$ is added to a solution containing $D$ - or $L$-Cys/Au NPs, one observes an increase in the rotation of polarized light as the concentration of racemic PO is increased. The increases in rotation angle are equal but of opposite sign for solutions with $D$ - and $L$-Cys/Au NPs. Although the racemic PO itself does not rotate light at any concentration, its enantiospecific interaction with the chiral Au NPs results in optical rotation at all concentrations. This arises because of two effects: the preferential adsorption of one enantiomer of PO onto the $D$ - or $L$-Cys/Au NPs and a change in the specific optical rotation of light by PO as a result of adsorption onto the Au NPs. More recently, we have shown that careful measurement of the optical rotation as a function of the enantiomeric excess of the PO and as a function of the enantiomeric excess of the $D$ - and $L$-Cys/Au NPs can be used to extract quantitative estimates of the enantiospecificity of the adsorption equilibrium constants for $R$ - and $S$-PO on $D$ - and $L$ Cys/Au NPs, $K_{D}^{R} / K_{L}^{R}=K_{L}^{S} / K_{D}^{S} \neq 1^{6}$.

In this work, we demonstrate the potential for use of chiral THH-Au NPs as adsorbent phases. The Au-NPs used in prior work were roughly spherical in shape and $\sim 5 \mathrm{~nm}$ in diameter, exposing a wide range of different surface structures. The local surface structures of the NPs can be chiral, if they have structures lacking mirror symmetry ${ }^{11-12}$ and can influence the enantioselectivity of adsorption; however, on the spherical NPs the inhomogeneous distribution of different surface orientations averages their influence on enantioselectivity to zero. In this work we use THH NPs with surface facets of well-defined, but achiral orientations. THH Au NPs have 24 facets and can be synthesized with high Miller index facets such as [037], [520], 
[210], and [740] ${ }^{13-17}$. Au NPs with high-index planes can have high densities of atoms or steps which result in high surface energy. In comparison with low Miller index planes such [111], [100] and [110], the high index planes show higher chemical activity and selectivity.

In addition to the advance of using single crystalline Au NPs, the work described herein demonstrates that the optical polarimetry method can be used to detect and study the enantiospecific adsorption of a known pharmaceutical, PLL, rather than simple chiral probe molecules such as PO.

\section{Experimental}

\subsection{Synthesis of THH-Au NPs}

Synthesis of THH-Au NPs was conducted according to the procedure developed by Ming et

$a l^{13}$. A typical synthesis procedure begins with preparation of a $\mathrm{HAuCl}_{4} / \mathrm{CTAB}$ seed solution. This begins by adding $0.25 \mathrm{~mL}$ of $0.01 \mathrm{M} \mathrm{Au}^{\mathrm{III}}$ chloride trihydrate $\left(\mathrm{HAuCl}_{4} \cdot 3 \mathrm{H}_{2} \mathrm{O}\right)$ solution to $9.75 \mathrm{~mL}$ of $0.1 \mathrm{M}$ cetyl trimethylammonium bromide (CTAB) solution at $37^{\circ} \mathrm{C}$ while stirring. Next, a $0.6 \mathrm{~mL}$ aliquot of $0.01 \mathrm{M}$ sodium borohydride $\left(\mathrm{NaBH}_{4}\right)$ solution was quickly injected into the $\mathrm{HAuCl}_{4} / \mathrm{CTAB}$ solution. The resulting solution was rapidly stirred for 1 minute then covered and left to rest for $3 \mathrm{hrs}$ at room temperature. This solution was placed in a hot water bath at $37^{\circ} \mathrm{C}$ to dissolve any CTAB crystals that had precipitated. Finally, a 1:50 dilution of the solution with deionized water yielded the $\mathrm{HAuCl}_{4} / \mathrm{CTAB}$ seed solution.

The THH-Au NP growth solution was prepared by adding $40 \mathrm{~mL}$ of $0.1 \mathrm{M} \mathrm{CTAB}$ to $2.0 \mathrm{~mL}$ of $0.01 \mathrm{M} \mathrm{HAuCl}_{4}$ while gently stirring. Next $0.4 \mathrm{~mL}$ of $0.01 \mathrm{M}$ silver nitrate $\left(\mathrm{AgNO}_{3}\right), 0.8 \mathrm{~mL}$ of $1.0 \mathrm{M} \mathrm{HCl}$, and $0.32 \mathrm{~mL}$ of $0.1 \mathrm{M}$ ascorbic acid were added in that order. After addition of ascorbic acid the color changes from orange to colorless. At this point, a $250 \mu \mathrm{L}$ aliquot of dilute $\mathrm{HAuCl}_{4} / \mathrm{CTAB}$ seed solution was quickly added to create the THH-Au NP growth solution. The growth solution was gently stirred for an additional 3 seconds then covered with Parafilm ${ }^{\mathrm{TM}}$ and left to rest for 8 hours to allow the seeds to grow into THH-Au NPs. The final color of the THH-Au NP solution was purple-brown. 


\subsection{Coating of THH-Au NPs with D or L-cysteine}

In order to chirally modify the THH-Au NPs, their surfaces were coated with $D$ - or $L$-Cys by mixing $1 \mathrm{~mL}$ of the THH-Au NP solution with $4 \mathrm{~mL}$ of $0.050 \mathrm{M} D$ - or $L$-Cys in deionized water 6-8. The solution was sonicated for approximately 30 minutes and then allowed to sit for approximately one hour before making optical rotation measurements.

\subsection{Characterization of THH-Au NPs}

The size, shape and morphology of THH-Au NPs were examined by SEM (scanning electron microscopy) and TEM (transmission electron microscopy). In order to prepare a sample of THH-Au NPs for SEM and TEM, a $1 \mathrm{~mL}$ solution of THH-Au NPs was centrifuged at 10,000 rpm for 5 min to separate the THH-Au NPs from the solution. The supernatant liquid was discarded and the remaining THH-Au NP precipitate was suspended in $50 \mu \mathrm{L}$ of water. $10 \mu \mathrm{L}$ of this THH-Au NP solution was drop-cast onto a carbon coated Cu TEM grid and the sample was dried for more than $24 \mathrm{hrs}$. The high resolution TEM studies were conducted using a Technai F20 FEG/HRTEM/ STEM with a Gatan imaging filter and Energy Dispersive X-ray spectroscopy operating at $200 \mathrm{keV}$. SEM images were obtained using a FEI Helios PFIB 660 with an L-Star electron column. The instrument operates at a voltage of $5 \mathrm{kV}$ and a current of $5.4 \mathrm{pA}$.

UV-vis spectrophotometry was used to compare the THH-Au NPs synthesized to those produced by Ming et al ${ }^{13}$. The THH-Au NP solution was diluted by a factor of two with DI water and scanned over the range 400 to $900 \mathrm{~nm}$ using a Cary UV-vis spectrophotometer.

A Rudolph Research Analytical Autopol VI was used for the optical polarimetry measurements. This has the ability to control the temperature of the sample and to measure optical rotations at six different wavelengths. A typical optical rotation measurement involved putting $3 \mathrm{~mL}$ of solution in the TempTrol polarimeter sample cell. For each sample, ten measurements were taken at a wavelength of $436 \mathrm{~nm}$ and a temperature of $23^{\circ} \mathrm{C}$. The average of these ten values was reported.

In order to estimate the values of the specific optical rotation constants for $R$ - and $S$-PLL, $\alpha_{\text {sol }}^{R}=-\alpha_{\text {sol }}^{S}$, solutions of $R$ - and $S$-PLL were made by dissolving $0.01 \mathrm{~g}$ of $R$-PLL or $S$-PLL in $5 \mathrm{~mL}$ of deionized water. The solution was then sonicated for $1 \mathrm{~min}$ and the optical rotation was measured. This process was repeated using four more $0.01 \mathrm{~g}$ additions of $R$-PLL or $S$-PLL to 



Figure 1. A) SEM image of THH-Au NPs taken before surface modification by Cys and showing rod-like morphology. B) HRTEM image of the THH-Au NPs. C) High resolution image revealing the (100) planes. D) Molecular structure of propranolol (PLL).

produce two plots of optical rotation angle versus concentration. The slope of the best fit line for each plot was used for $\alpha_{\text {sol }}^{R}$ or $\alpha_{\text {sol }}^{S}$.

$\mathrm{Au}^{\mathrm{III}}$ chloride trihydrate (>99.9\%), sodium borohydride, $L$-cysteine (>97\%), $D$-cysteine (99\%), and $D L$-cysteine (>97\%), cetyl trimethylammonium bromide, silver nitrate (99\%), ascorbic acid (99\%), $R$ - and $S$-propranolol hydrochloride (>98\%) were purchased from Sigma Aldrich and were used without further purification.

\section{Results}

\subsection{THH-Au NP characterization}

The THH-AU NPs were characterized initially using SEM, TEM and UV-vis spectrophotometry. The SEM image in Figure 1A reveals the formation of Au NPs with a highly homogeneous morphology. The NPs are rod-like with $\sim 40 \mathrm{~nm}$ diameter and lengths of $\sim 100 \mathrm{~nm}$. The bright field TEM images of Figure 1B\&C reveal that the NPs are crystalline with wellformed facets. The planes revealed by the high resolution image in Figure 1C are spaced along the [100] direction. Although the facets orientation have not been determined in this work, prior work shows that they are achiral, high Miller index planes ${ }^{13-17}$. The UV-vis spectra are also 


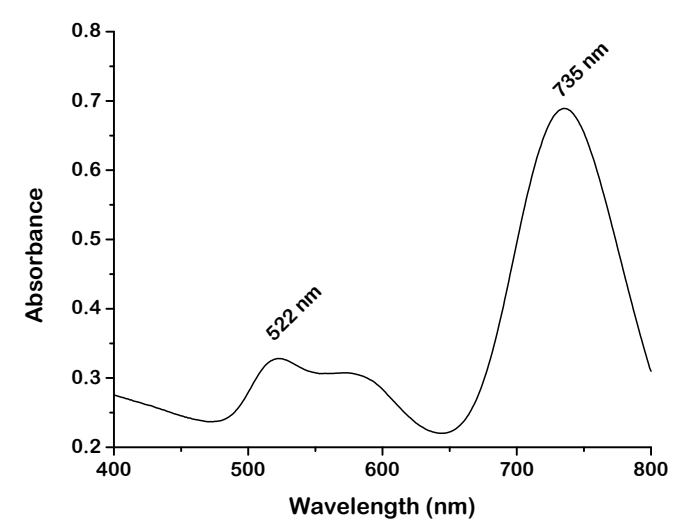

Figure 2. UV-vis spectrum of THH-Au NPs in solution. The two primary peaks at $522 \mathrm{~nm}$ and $735 \mathrm{~nm}$ are characteristic of Au NPs.

characteristic of THH-Au NPs. Figure 2 reveals two distinct absorption peaks at $522 \mathrm{~nm}$ and $735 \mathrm{~nm}$ characteristic of Au NPs ${ }^{13,18}$.

\subsection{Optical rotation by PLL in Cys/THH-Au NPs}

The enantiospecific interaction of $R$ - and $S$-PLL with $D$ - and $L$-Cys/THH-Au NPs has been studied quantitatively using optical polarimetry to measure the rotation of linearly polarized light passing through solutions containing mixtures of $R$ - and $S$-PLL with varying enantiomeric excess and either $D$ - or $L$-Cys/THHAu NPs. Alternatively, we have also measured the rotation of linearly polarized light passing through solutions containing either pure $R$ - or pure $S$-PLL and mixtures of $D$ - and $L$ Cys/THH-Au NPs with varying enantiomeric excess. Figure 3 shows a schematic representation of the measurement protocol implemented to prepare solutions with well-controlled fractional concentrations of $R$ - and $S$-PLL, defined as $\beta=x^{R} /\left(x^{R}+x^{S}\right)$, in solutions with constant concentrations of $D$ - or $L$-Cys/THHAu NPs. The complementary protocol was used to prepare solutions with well-controlled fractional concentrations of $D$ - and $L$-Cys/THH-Au NPs, defined as $\gamma=x^{L} /\left(x^{D}+x^{L}\right)$, in solution

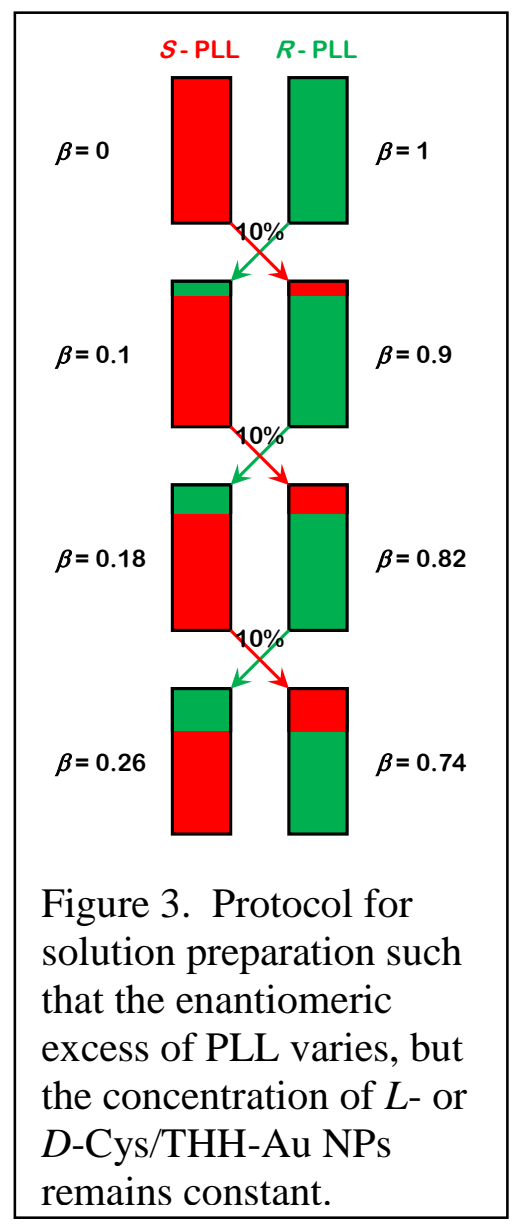


A



B

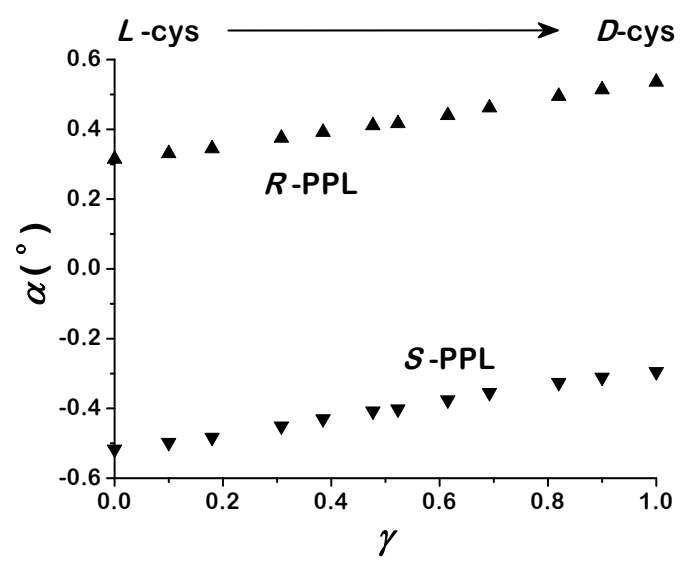

Figure 4. A) Optical rotation angles measured for solutions containing constant concentrations of $D$ - or $L$-Cys/THH-Au NPs $\left(1.1 \times 10^{14} \mathrm{NP} / \mathrm{ml}\right)$ and mixtures of $R$ - and $S$-PLL with varying values of $\beta$ but total concentration of $0.05 \mathrm{M}$. B) Optical rotation angles measured for solutions containing constant concentrations of $R$ - or $S$-PLL $(0.05 \mathrm{M})$ and mixtures of $D$ - or $L$-Cys/THH-Au NPs $\left(1.1 \times 10^{14} \mathrm{NP} / \mathrm{ml}\right)$ with varying values of $\gamma$. Note that solutions exhibit diastereomerism in that the solutions with opposite chirality have optical rotations with equal but opposite sign.

with constant concentrations of $0.05 \mathrm{M} R$ - or $S$-PLL. In the case of the measurements that spanned $\beta=0 \rightarrow 1$, two $5 \mathrm{ml}$ aqueous solutions were prepared initially with identical concentrations of $D$-Cys/THH-Au NPs and $0.05 \mathrm{M}$ of $R$-PLL and $S$-PLL in each. After measuring the optical rotation by these two solutions having $\beta=0$ and $\beta=1$, aliquots of $0.5 \mathrm{ml}$ were transferred between them. Optical rotation measurements were then made using these solutions now having $\beta=0.1$ and $\beta=0.9$. Then $0.5 \mathrm{ml}$ aliquots were transferred between the two and another set of optical rotation measurements was made. This transferring of solution between vials was continued until $\beta$ approached 0.5 for both the solutions. A similar protocol was used for the measurements in which the fractional concentrations of $D$ - and $L$-Cys/THH-Au NPs were varied from $\gamma=0 \rightarrow 1$.

Figure 4A plots the optical rotation angles measured for solutions containing $D$ - or $L$ Cys/THH-Au NPs $\left(1.1 \times 10^{14} \mathrm{NP} / \mathrm{ml}\right)$ as functions of $\beta$ with a total PLL concentration of $0.05 \mathrm{M}$. These data are linear in $\beta$, as expected ${ }^{7}$. Note that solutions of opposite chirality $(D-C y s / A u$ at $\beta$ versus $L-\mathrm{Cys} / \mathrm{Au}$ at $1-\beta$ ) exhibit optical rotation angles that are equal in magnitude but opposite in sign. They are related by diastereomerism. Figure 4B plots the optical rotation angles for solutions containing $R$-PLL or $S$-PLL at a constant concentration of $0.05 \mathrm{M}$ as a function of $\gamma$. 
The total concentration of Au NPs in these solutions is estimated at $1.1 \times 10^{14} \mathrm{NP} / \mathrm{ml}$. These data also exhibit diastereomerism. Details of the adsorption behavior and the model used to quantify these data are given in the Discussion section.

Perhaps the clearest demonstration of the enantiospecific interaction of the PLL with the chirally modified THH-Au NPs comes from measurement of optical rotation of light during addition of racemic $R S$-PLL to solutions containing $D$ - or $L$ -

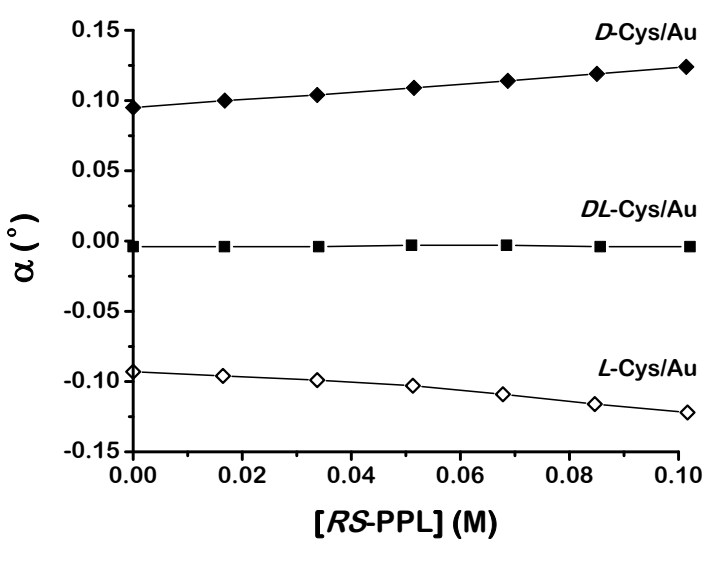

Figure 5. Optical rotation of light by addition of $R S$-PLL to solutions containing THH Au NPs coated with $D-, L-$, or $D L$-Cys. Addition to solutions with $D L-\mathrm{Cys} / \mathrm{Au}$ shows no rotation Cys/THH-Au NPs (Figure 5). Figure 5

shows the control experiments, optical rotation by solutions containing racemic $D L-\mathrm{Cys} / \mathrm{THH}-$ $\mathrm{Au}$ NPs and various concentrations of $R S$-PLL. As expected, $D L-\mathrm{Cys} / \mathrm{THH}-\mathrm{Au}$ NPs alone induce no optical rotation ( $\boldsymbol{\square}$ ) and the addition of $R S$-PLL induces no rotation of light. Addition of $R S$-PLL to the solution containing pure $D$-Cys/THH-Au NPs induced a rotation of $+0.09 \% \mathrm{M}$ $(\diamond)$ while addition to the solution containing pure $L$-Cys/THH-Au NPs induced a rotation of $0.09^{\circ} / \mathrm{M}(\diamond)$. As shown in previous work, the optical rotation during addition of a racemic mixture to a solution of chiral NPs arises, in part, as a result of the enantiospecific interactions between PLL enantiomers and the chiral THH-Au NPs resulting in enantioselective adsorption and enantiospecific partitioning between the solution phase and the adsorbed phase. This is observable using optical polarimetry because adsorption onto the Au NPs also results in a change in the specific optical rotation of light by the $\mathrm{PLL}^{7}$.

\section{Discussion}

\subsection{Adsorption and optical rotation model}

In previous work, a model was developed from a Langmuir adsorption isotherm to relate optical rotation measurements to the physical processes and parameters governing adsorption of chiral molecules on chiral Au NPs ${ }^{6-7}$. Figure 6 illustrates the adsorption equilibria that determine the relative concentrations of $R$ - and $S$-PLL in the solution phase and adsorbed on the 
$D$ - and $L$-Cys/THH-Au NPs. The total concentrations of Cys/THH-Au NPs and PLL are assumed to be constant. Two terms, $\beta$ and $\gamma$, define the fractional compositions of a given solution. The value $\beta=0 \rightarrow 1$ refers to the fractional concentration of $R$-PLL in solutions containing both PLL enantiomers.

$$
\begin{aligned}
& x_{t o t}^{R}=x_{s o l}^{R}+x_{a d s}^{R}=\beta \cdot x_{t o t}^{P L L} \\
& x_{t o t}^{S}=x_{s o l}^{S}+x_{a d s}^{S}=(1-\beta) \cdot x_{t o t}^{P L L}
\end{aligned}
$$

The quantity $x_{t o t}^{R}$ is the concentration of $R$-PLL with units of M and $x_{t o t}^{S}$ is the concentration of $S$-PLL, and $x_{t o t}^{P L L}$ is the total concentration of PLL. A similar term, $\gamma=0 \rightarrow 1$, defines the fraction of $L$-Cys/THH-Au NPs in a solution containing both NP enantiomers.

$$
\begin{aligned}
& x_{t o t}^{D}=\gamma \cdot x_{t o t}^{A u} \\
& x_{t o t}^{L}=(1-\gamma) \cdot x_{t o t}^{A u}
\end{aligned}
$$

An equilibrium constant, $K$, with units of $\left(\mathrm{M}^{-1}\right)$ is assigned to each of the four diastereomeric PLL-Cys/THH-Au NP combinations. For instance, $K_{D}^{R}$ is the equilibrium constant governing adsorption of $R$-PLL onto the surfaces of $D$-Cys/THH-Au NPs. Diastereomerism, constrains these four equilibrium constants to having the following relationships: $K_{D}^{R}=K_{L}^{S} \neq K_{D}^{S}=K_{L}^{R}$.

The quantities $\alpha$ are the specific optical rotation constants for PLL in units of $(\% / M)$. A critical point is that $\alpha_{\text {sol }} \neq \alpha_{a d s}$ and, as a consequence, the net rotation of light by a solution containing $R$-PLL is determined by the fraction in the solution phase and the fraction in the adsorbed phase. The dimensionless parameter

$$
\bar{\alpha}=\alpha_{\text {ads }}^{R} / \alpha_{\text {sol }}^{R}=\alpha_{\text {ads }}^{S} / \alpha_{\text {sol }}^{S}
$$

quantifies the ratio between the specific optical rotation constants of PLL in the adsorbed and solution phases. The values of $\alpha_{\text {sol }}^{R}=-\alpha_{\text {sol }}^{S}$ are readily determined from measurements of optical rotation by solutions of $R$-PLL and $S$-PLL. However, the value of $\alpha_{a d s}^{R}=-\alpha_{a d s}^{S}$ cannot 


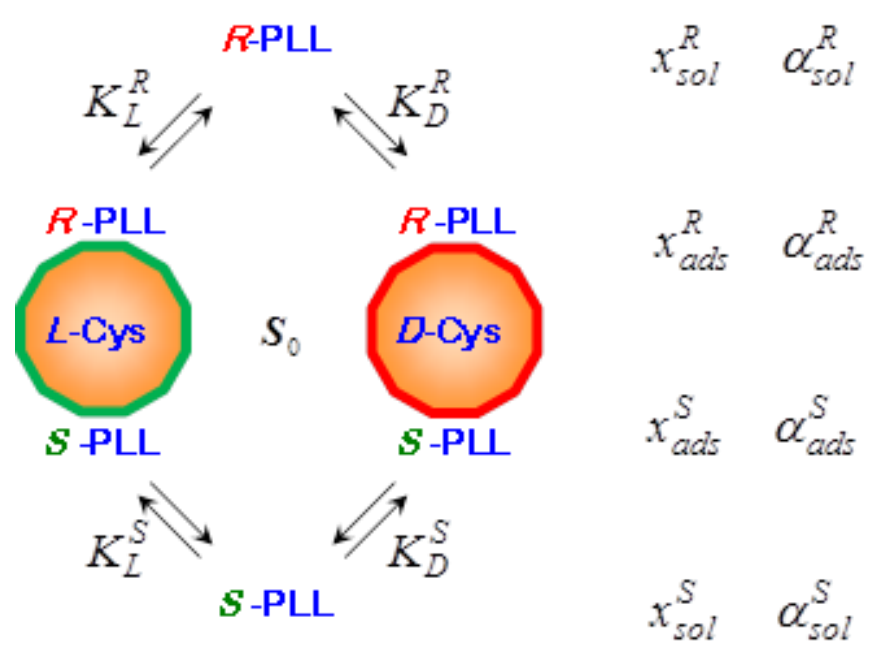

Figure 6. The physical phenomena and the parameters governing optical rotation by solutions of $R$ - and $S$-PLL with $D$ - and $L$-Cys/THH-Au NPs. The equilibria between the concentrations of PLL in the solution phase, $x_{\text {sol }}$, and in the adsorbed phase, $x_{a d s}$, are dictated by the equilibrium constants, $K$. The quantity, $S_{0}$, represents the concentration of PLL adsorption sites on the Cys/THH-Au NPs. The specific optical rotation constants, $\alpha$, for PLL differ in the adsorbed and solution phases. Diastereomerism gives the following relationships between quantities: $K_{D}^{R}=K_{L}^{S} \neq K_{L}^{R}=K_{D}^{S}, \alpha_{\text {ads }}^{R}=-\alpha_{\text {ads }}^{S}$ and $\alpha_{\text {sol }}^{R}=-\alpha_{\text {sol }}^{S}$.

be determined easily. Therefore, $\bar{\alpha}$ is an unknown parameter that must be extracted from measurements of optical rotation by solutions containing both PLL and Cys/THH-Au NPs.

The quantity $S_{0}$ represents the concentration in solution of PLL binding sites on the Cys/THH-Au NPs. This value is also difficult to measure; however, under conditions where the coverage of PLL on the Cys/THH-Au NPs is low, the adsorption isotherms depend only on the coupled quantities

$$
\begin{aligned}
& \bar{K}_{D}=S_{0} K_{D}^{R}=S_{0} K_{L}^{S} \\
& \bar{K}_{L}=S_{0} K_{D}^{S}=S_{0} K_{L}^{R}
\end{aligned}
$$

which can also be extracted from measurements of optical rotation by solutions containing both PLL and Cys/THH-Au NPs.

Optical rotation measurements have been made using the two protocols described in Sec. 3.2 for varying $\beta$ and $\gamma$. One is indicated by Figure 4A which shows the optical rotation measured using a constant concentration of either $D$ - or $L$-Cys/THH Au NPs $\left(1.1 \times 10^{14} \mathrm{NP} / \mathrm{ml}\right)$ with varying enantiomer fraction, $\beta$, of $R$ - and $S$-PLL at a constant total concentration of $0.05 \mathrm{M}$. The 


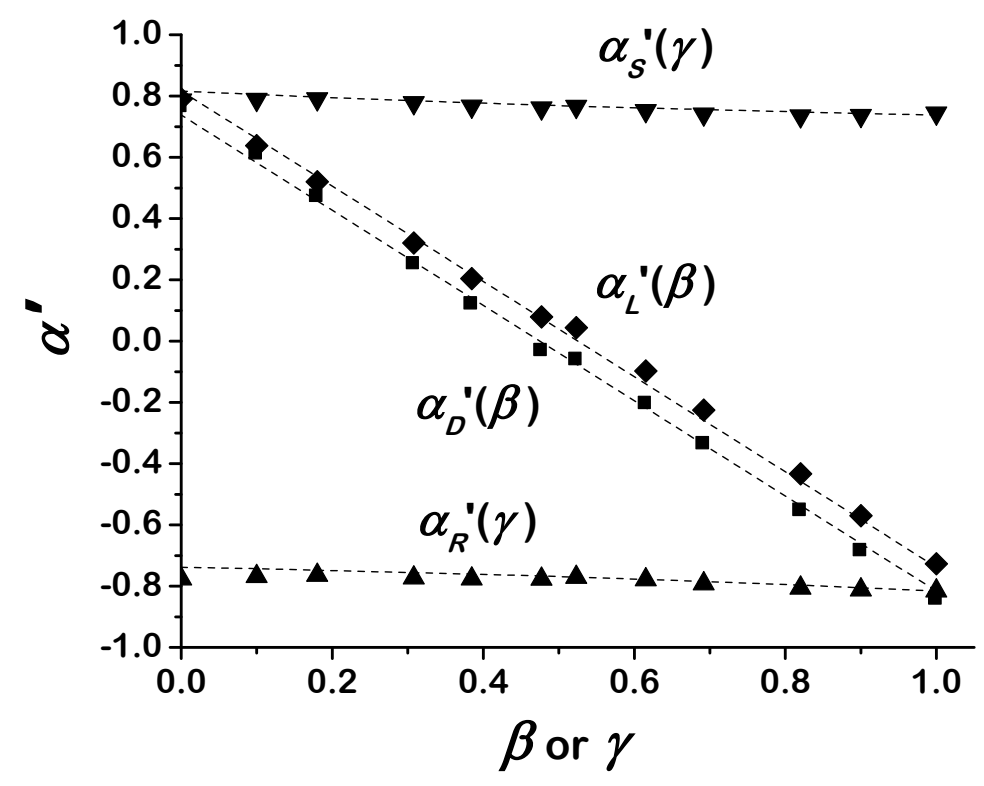

Figure 7. Dimensionless optical rotation measurements from Figure 4 plotted versus $\beta$ and $\gamma$. The curves are the optimal fits of equations 9 and 10 to the data using $\bar{\alpha}, \bar{K}_{D}$, and $\bar{K}_{L}$ as fitting parameters.

optical rotation measurements obtained using the other protocol are found in Figure 4B which shows the optical rotation measured using a constant concentration of $0.05 \mathrm{M} R$ - or $S$-PLL with varying enantiomer fraction, $\gamma$, of $D$ - and $L$-Cys/THH Au NPs at a constant total concentration of $1.1 \times 10^{14} \mathrm{NP} / \mathrm{ml}$. The optical rotations, $\alpha$, measured from these solutions have been rendered dimensionless using

$$
\alpha^{\prime}=\frac{\alpha}{x_{\text {tot }}^{P L L} \cdot \alpha_{\text {sol }}^{S}}
$$

and are replotted in Figure 7.

Under conditions in which the coverage of PLL on the Cys/THH-Au NP surfaces is low, the equilibrium coverage will be linear in the solution phase concentration. The data illustrated in Figure 4 have been obtained in this regime. Under these conditions, the dimensionless optical rotations can be expressed using equations 9 and 10. Note that, unfortunately, these expressions were printed incorrectly in reference ${ }^{6}$ and are therefore corrected here. 


$$
\begin{aligned}
& \alpha_{D}^{\prime}(\beta)=\left(\frac{1+\bar{\alpha} \bar{K}_{L}}{1+\bar{K}_{L}}\right)-\left[\left(\frac{1+\bar{\alpha} \bar{K}_{L}}{1+\bar{K}_{L}}\right)+\left(\frac{1+\bar{\alpha} \bar{K}_{D}}{1+\bar{K}_{D}}\right)\right] \beta \\
& \alpha_{L}^{\prime}(\beta)=\left(\frac{1+\bar{\alpha} \bar{K}_{D}}{1+\bar{K}_{D}}\right)-\left[\left(\frac{1+\bar{\alpha} \bar{K}_{L}}{1+\bar{K}_{L}}\right)+\left(\frac{1+\bar{\alpha} \bar{K}_{D}}{1+\bar{K}_{D}}\right)\right] \beta \\
& \alpha_{S}^{\prime}(\gamma)=\frac{1+\bar{\alpha}\left(\gamma \bar{K}_{L}+(1-\gamma) \bar{K}_{D}\right)}{1+\left(\gamma \bar{K}_{L}+(1-\gamma) \bar{K}_{D}\right)} \\
& \alpha_{R}^{\prime}(\gamma)=-\frac{1+\bar{\alpha}\left(\gamma \bar{K}_{D}+(1-\gamma) \bar{K}_{L}\right)}{1+\left(\gamma \bar{K}_{D}+(1-\gamma) \bar{K}_{L}\right)} .
\end{aligned}
$$

The values of the three parameters $\bar{\alpha}, \bar{K}_{D}$, and $\bar{K}_{L}$ have been estimated by fitting equations 9 and 10 to the data in Figure 7 . The curves in Figure 7 represent the best fits to the experimental data and yield values of $\bar{\alpha}=0.59, \bar{K}_{L}=1.73$, and $\bar{K}_{D}=0.80$. Figure 8 plots the sum of squared errors use to estimate the parameters $\bar{\alpha}, \bar{K}_{D}$, and $\bar{K}_{L}$ evaluated at $\bar{\alpha}=0.59$ and illustrates the regions of $66 \%$ and $95 \%$ confidence in the values of $\bar{K}_{D}$, and $\bar{K}_{L}$. The key point is that $\bar{K}_{D}$ and $\bar{K}_{L}$ are significantly different and, therefore, reveal true enantiospecific adsorption of PLL on chiral Cys/THH-Au NPs with $\bar{K}_{L} / \bar{K}_{D}=2.2$. Note that this value is similar to others measured in for PO adsorption on chiral Au NPs and for enantiospecific adsorption of chiral probe molecules on chiral single crystal surfaces ${ }^{6-7,19}$.

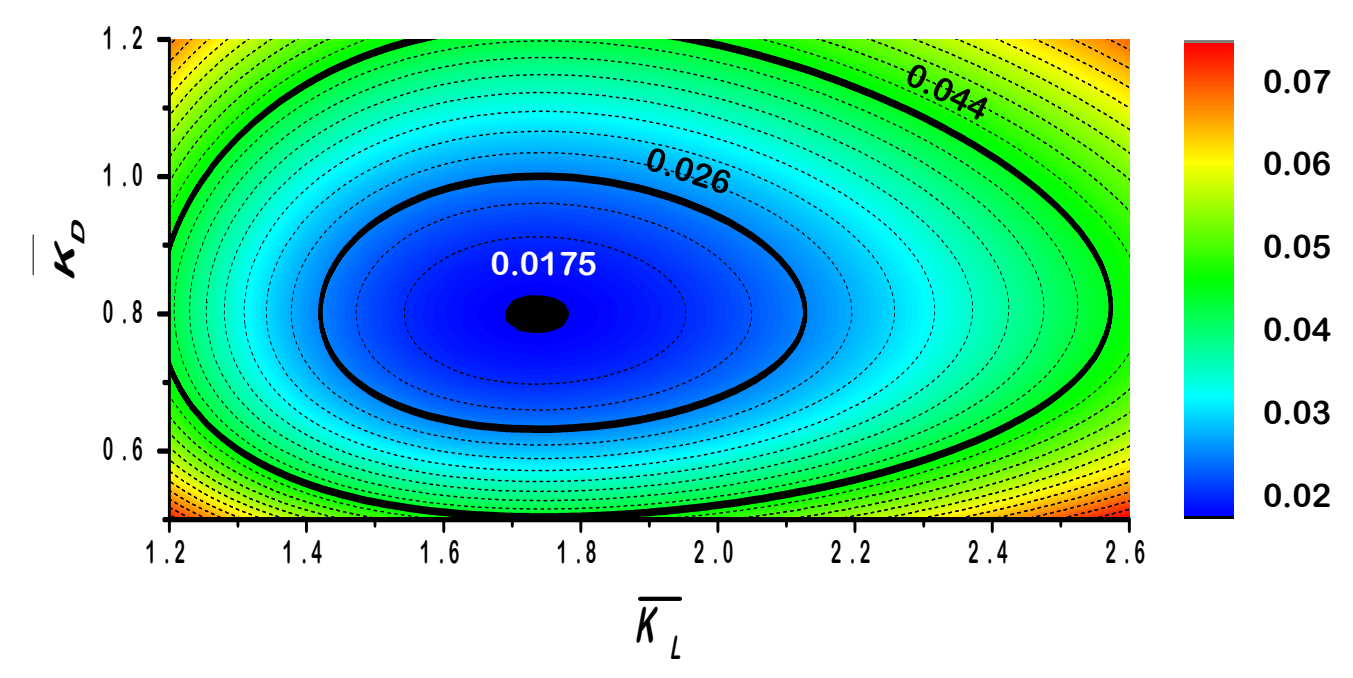

Figure 8. Plot of the sum of squared errors (SSE) objective function used to fit equations 9 and 10 to the data in Figure 7. The value of SSE is plotted versus $\bar{K}_{D}$, and $\bar{K}_{L}$ evaluated at $\bar{\alpha}=0.59$. The contour at SSE $=0.026$ estimates the region of $\pm 1 \sigma$ confidence and the contour at $\mathrm{SSE}=0.44$ estimates the region of $\pm 2 \sigma$ confidence. 


\section{Conclusions}

The binding of $R$ - or $S$-PLL from aqueous solution to the chirally modified surfaces of $D$ and $L$-Cys/THH-Au NPs is enantiospecific. In particular, the equilibrium constant for adsorption of $R$-PLL to the surfaces of $L$-Cys/THH-Au NPs is 2.2 times that of $S$-PLL. By diastereomerism the binding of $S$-PLL to the surfaces of $L$-Cys/THH-Au NPs is 2.2 times that of $R$-PLL. These enantiospecific interactions with chiral Au NPs could be used as the basis for separations methods based on chromatography or centrifugation.

\section{Acknowledgements}

This work has been funded by the US DOE through grant number DE-FG02-12ER16330. 


\section{References}

1. Bingyun, L.; Haynie, D., Chiral Drug Separation In Encyclopedia of Chemical Processes, Lee, S., Ed. Taylor and Francis: 2006; Vol. 1, pp 449-458.

2. Nguyen, L. A.; He, H.; Pham-Huy, C., Chiral Drugs: An Overview. International journal of biomedical science : IJBS 2006, 2 (2), 85-100.

3. Dolamic, I.; Knoppe, S.; Dass, A.; Buergi, T., First Enantioseparation and Circular Dichroism Spectra of Au-38 Clusters Protected by Achiral Ligands. Nature Communications 2012, 3.

4. Knoppe, S.; Dolamic, I.; Dass, A.; Buergi, T., Separation of Enantiomers and Cd Spectra of Au40(Sch2ch2ph)24: Spectroscopic Evidence for Intrinsic Chirality. Angewandte ChemieInternational Edition 2012, 51 (30), 7589-7591.

5. Su, H.; Zheng, Q.; Li, H., Colorimetric Detection and Separation of Chiral Tyrosine Based on NAcetyl-L-Cysteine Modified Gold Nanoparticles. Journal of Materials Chemistry 2012, 22 (14), 6546-6548.

6. Shukla, N.; Ondeck, N.; Gellman, A. J., Quantitation of Enantiospecific Adsorption on Chiral Nanoparticles from Optical Rotation. Surf. Sci. 2014, 629, 15-19.

7. Shukla, N.; Bartel, M. A.; Gellman, A. J., Enantioselective Separation on Chiral Au Nanoparticles. J. Amer. Chem. Soc. 2010, 132 (25), 8575-8580.

8. Shukla, N.; Ondeck, N.; Khosla, N.; Klara, S.; Petti, A.; Gellman, A., Polarimetric Detection of Enantioselective Adsorption by Chiral Au Nanoparticles - Effects of Temperature, Wavelength and Size. Nanomaterials and Nanotechnology 2015, 5, 1-8.

9. Chen, L.; Ma, H.; Liu, X.; Jiang, S. x., Semipreparative Enantiomer Seperation of Propranolol Hydrochloride by High-Performance Liquid Chromatography Using Cellulose Tris(3,5Dimethylphenylcarbamate) Chiral Stationary Phase. Journal of Chromatographic Science 2008, 46 (9), 767-771.

10. Jadzinsky, P. D.; Calero, G.; Ackerson, C. J.; Bushnell, D. A.; Kornberg, R. D., Structure of a Thiol Monolayer-Protected Gold Nanoparticle at 1.1 Angstrom Resolution. Science 2007, 318 (5849), 430433.

11. McFadden, C. F.; Cremer, P. S.; Gellman, A. J., Adsorption of Chiral Alcohols on "Chiral" Metal Surfaces. Langmuir 1996, 12 (10), 2483-2487.

12. Horvath, J. D.; Koritnik, A.; Kamakoti, P.; Sholl, D. S.; Gellman, A. J., Enantioselective Separation on a Naturally Chiral Surface. J. Amer. Chem. Soc. 2004, 126 (45), 14988-14994.

13. Ming, T.; Feng, W.; Tang, Q.; Wang, F.; Sun, L.; Wang, J.; Yan, C., Growth of Tetrahexahedral Gold Nanocrystals with High-Index Facets. J. Amer. Chem. Soc. 2009, 131 (45), 16350-+.

14. Su, D.; Dou, S.; Wang, G., Gold Nanocrystals with Variable Index Facets as Highly Effective Cathode Catalysts for Lithium-Oxygen Batteries. Npg Asia Materials 2015, 7.

15. Kim, D. Y.; Im, S. H.; Park, O. O., Synthesis of Tetrahexahedral Gold Nanocrystals with High-Index Facets. Crystal Growth \& Design 2010, 10 (8), 3321-3323. 
16. Li, J.; Wang, L.; Liu, L.; Guo, L.; Han, X.; Zhang, Z., Synthesis of Tetrahexahedral Au Nanocrystals with Exposed High-Index Surfaces. Chemical Communications 2010, 46 (28), 5109-5111.

17. Yin, P.-G.; You, T.-T.; Tan, E.-Z.; Li, J.; Lang, X.-F.; Jiang, L.; Guo, L., Characterization of Tetrahexahedral Gold Nanocrystals: A Combined Study by Surface-Enhanced Raman Spectroscopy and Computational Simulations. Journal of Physical Chemistry C 2011, 115 (37), 18061-18069.

18. Amendola, V.; Meneghetti, M., Size Evaluation of Gold Nanoparticles by Uv-Vis Spectroscopy. Journal of Physical Chemistry C 2009, 113 (11), 4277-4285.

19. Yun, Y. J.; Gellman, A. J., Enantioselective Separation on Naturally Chiral Metal Surfaces: D,LAspartic Acid on $\mathrm{Cu}(3,1,17)(\mathrm{R} \& S)$ Surfaces. Angew. Chem.-Int. Edit. 2013, 52 (12), 3394-3397. 


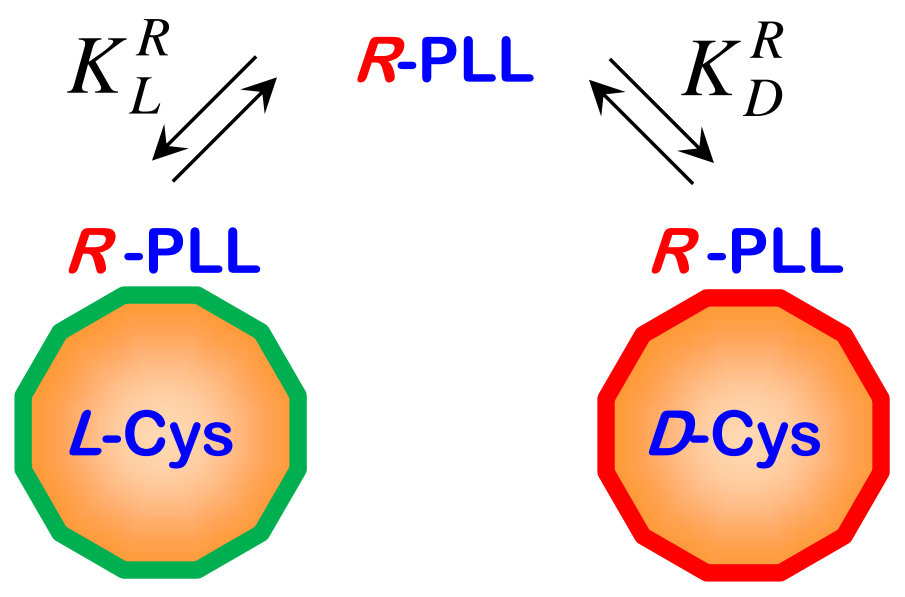

